\title{
LA CREACIÓN ARTÍSTICA EN LA EDUCACIÓN DE LAS PERSONAS CON DIVERSIDAD FUNCIONAL: UNA INVESTIGACIÓN-ACCIÓN ${ }^{1}$
}

\author{
Recepción: 19/02/2019 | Revisión: 04/03/2019 | Aceptación: 12/09/2019
}

\author{
Mar MORÓN VELASCO \\ Universitat Autònoma de Barcelona \\ mar.moron@uab.cat
}

\begin{abstract}
Resumen: El siguiente texto presenta una investigación-acción sobre cómo los espacios de creación artística, espacios de libertad con seguridad, en un contexto educativo con niños con diversidad funcional (DF), desarrollan los componentes de una conducta auto-determinada. Una primera parte recorre dos campos de estudio que generalmente van en paralelo: la creación artística y la educación de las personas con DF para, en una segunda parte, enlazarlos en una experiencia de intervención educativa, objeto de análisis y reflexión. La investigación-acción realizada demuestra cómo la creación artística favorece que los alumnos con DF actúen de una manera autónoma, que auto-regulen sus conductas, que inicien y respondan a los acontecimientos de una manera psicológicamente hábil y, por último, que la persona actúe conscientemente.
\end{abstract}

Palabras clave: creación artística; diversidad funcional; investigación-acción.

\section{ARTISTIC CREATION INTHE EDUCATION OF PEOPLE WITH FUNCTIONAL DIVERSITY: AN ACTION- RESEARCH PROJECT}

\begin{abstract}
The following text presents an action-research project on how spaces of artistic creation, spaces of freedom with security, in an educational context with children with functional diversity (FD), develop the components of a selfdetermined behaviour. The first part covers two fields of study which generally run in parallel: the artistic creation and the education of people with FD. Secondly, it relates these people to an experience of educational intervention, object of analysis and reflection of this paper. This action-research project demonstrates how artistic creation leads students with DF to acting autonomously, to self-regulating their behaviour, to initiating and responding to events in a psychologically proficient manner and, finally, to acting consciously.
\end{abstract}

Keywords: artistic creation; functional diversity; actionresearch.

\section{LA CREACIÓ ARTISSTICA EN L'EDUCACIÓ DE LES PERSONES AMB DIVERSITAT FUNCIONAL: UNA INVESTIGACIÓ-ACCIÓ}

Resum: Aquest article presenta una investigació-acció sobre com els espais de creació artística, espais de llibertat amb seguretat, en un context educatiu amb nens amb diversitat funcional (DF), desenvolupen els components d'una conducta auto-determinada. Una primera part recorre dos camps d'estudi que generalment van en paral.lel: la creació artística i l'educació de les persones amb DF per, en una segona part, enllaçar-los en una experiència d'intervenció educativa, objecte d'anàlisi i reflexió. La investigació-acció realitzada demostra com la creació artística afavoreix que els alumnes amb DF actuïn d'una manera autònoma, que auto-regulin la conducta, que iniciön $i$ responguin als esdeveniments d'una manera psicològicament hàbil $i$, per últim, que la persona actuï conscientment.

Paraules clau: creació artística; diversitat funcional; investigacióacció.

1 Se puede encontrar más información sobre el tema en el capítulo del libro: Morón, M. (2012). "La importancia de la creación artística en la educación de las personas con discapacidad intelectual". En M. Olveira y C. Pérez (Coord.), Encuentro. Artes por la integración, pp. 75-87. A Coruña: Diputación de Galicia. Dipòsit legal. C 1564-2012 


\section{Introducción}

En el título de este texto, La creación artística en la educación de las personas con diversidad funcional: una investigación-acción, aparecen dos ámbitos educativos generales: por un lado, la educación de las Artes y, por otro, la educación de las personas con necesidades específicas. Aparecen también dos temáticas: la creación artística y las personas con diversidad funcional. Primeramente, el texto recorre una presentación teórica de estos temas para pasar a la parte empírica de este estudio: una investigación-acción llevada a cabo con 5 niños y una niña con diversidad funcional ${ }^{2}$ (DF).

Generalmente, encontramos estos dos ámbitos educativos separados: la educación artística y la educación de las personas con diversidad funcional ${ }^{3}$. Con este texto, intento romper ese camino paralelo para exponer conexiones entre ambos mundos y de qué manera se complementan, con el objetivo de mejorar la educación de un colectivo, a veces, en riesgo de exclusión social, y que merece las mismas oportunidades: las personas que necesitan de unos soportes específicos para alcanzar una buena calidad de vida.

Desde la posición de maestra e investigadora ${ }^{4}$, muestro el proceso y los resultados de esta investigación. Es un estudio sobre cómo las personas con diversidad funcional son capaces de adquirir unas competencias personales y sociales a través de espacios donde crear artísticamente de manera libre y segura, para conseguir ser personas auto-determinadas. Vamos describiendo cómo las personas son capaces de mejorar sus habilidades de auto-regulación, de auto-conocimiento, de percepción de control y eficacia, de establecer objetivos, de resolución de problemas, etc. (Wehmeyer, 2001) a partir de un proyecto de intervención educativa (con unas características que más tarde enumeraremos) basado en la creación artística.

El modelo de investigación-acción nos ha permitido conceptualizar la práctica como espacio de aplicación de la teoría, reflexionar sobre esa práctica y acabar teorizando sobre ella. La práctica educativa es considerada como punto de partida, como eje de formación, como objeto de reflexión y de construcción y, finalmente, como objeto de transformación. La práctica educativa se convierte en un lugar desde donde reflexionar para mejorar la educación: la escuela como laboratorio.

\section{La educación: la creación artística}

La primera intersección entre los dos ámbitos de trabajo de esta investigación es la educación. Educar comporta, para todas las personas, el esfuerzo de activar o potenciar todo aquello que la persona podrá dar de sí, hacer visibles sus capacidades, ampliar las posibilidades de ser y de estar a todos los niveles, lo que implica una tarea de observación, de ensayo y error, de seguimiento y de escucha

2 Utilizaremos el término de diversidad funcional para referirnos a personas con discapacidad intelectual.

3 Una cuestión que hace evidente esta afirmación es la poca formación que hay en los profesionales de estos dos ámbitos educativos. Si revisamos el grado de Bellas Artes difícilmente encontramos proyectos para personas con diversidad funcional, y si miramos la formación de los especialistas para una educación inclusiva, la presencia del área de educación artística es escasa. Resulta difícil encontrar profesionales formados en estos dos ámbitos.

4 La formación de la maestra-investigadora que relata esta investigación es en Educación Especial y en Bellas Artes, con amplia experiencia en ambos campos. 
constante de quien debe ser educado. Un seguimiento flexible, adaptado a cada individuo que, como tal, es único y diferente a cualquier otro. El educador/a o maestro/a funciona como modelo para sus educandos, por lo que será imprescindible un perfil de persona concreto, con unas características adecuadas a la tarea que realiza. Según García Mantilla (Ferrés, 2008:48), la educación implica favorecer el desarrollo integral de la persona partiendo de sus propias necesidades, apoyando su desarrollo físico y psíquico, permitiendo el ejercicio de todo un potencial de habilidades valiosas, sirviendo a una socialización que haga consciente al individuo de su papel en el mundo.

Consideramos la educación como un proceso de creación de nosotros mismos, para construirnos como personas críticas, responsables y activas, y que podamos formar parte de una sociedad democrática, progresista, equitativa, tolerante y diversa. Así, consideramos, que los niños y las niñas deben transitar experiencias de creación, aprender estrategias que les permitan llevar a cabo procesos donde se pregunten: qué quiero conseguir, cómo lo consigo, lo realizo y lo muestro. El profesor debe de ser acompañante de este proceso que significa el construirse como persona. La educación ha dejado de ser una acumulación de conocimientos que algunos han decidido que son verdaderos y necesarios, y valorados por una parte de la sociedad tradicionalista, conservadora, limitadora y manipuladora. Muy al contrario, debemos considerarla en función de la construcción de personas libres y críticas, creadoras de conocimiento y agentes causales de su vida.

La creación artística ha de estar presente en la formación de los individuos como forma de pensamiento, de percepción, de comprensión y de expresión; es un espacio íntimo de libertad y de conocimiento de uno mismo y del mundo, y un tiempo para expresar emociones, deseos, dudas y sentimientos por medio de los lenguajes artísticos. Posibilita un espacio de acercamiento al inconsciente, a sensaciones y pensamientos que se encuentran ocultos y que se hacen visibles, se formalizan mediante el proceso de creación artística que finaliza con la construcción del objeto artístico. En palabras de la filósofa Heike Freire (2008), la creación artística permite a cada persona la creación de sus propios símbolos, a partir de sus sensaciones y sentimientos, la educación artística promueve una sociedad de individuos con todas sus dimensiones (física, afectiva, intelectual, social y espiritual) integradas, vinculados en relación de igualdad, respeto y mutua satisfacción.

Definimos la creación artística como el proceso a través del cual la persona es capaz de generar un producto único/original (idea, artefacto, narrativa visual, etc.) que aparece desde su interior, desde sus experiencias y conocimientos, $y$ al que mediante un proceso/progreso, de conexión con el mundo, es capaz de darle forma y contenido, en un espacio de libertad y de encuentro con él mismo, con sus objetividades y subjetividades, y desde sus deseos y necesidades.

La experiencia creativa se desarrolla en un espacio-tiempo en el cual se expresan pensamientos, sentimientos, deseos y emociones, se perciben sensaciones, se transforma, se construye; en el que las personas se hacen conscientes de sus capacidades y de sus limitaciones desde las experiencias. Con la creación artística, se alcanza un conocimiento de uno mismo, de los otros y del entorno que permite una adaptación e implicación más fuerte con la vida; y también, desarrolla la inteligencia (desde un modelo multidimensional) en los ámbitos de la percepción, la memoria, la atención y el pensamiento. La creación artística es un modo de conocimiento, de expresión, de comunicación y de transformación. 
Si realizamos un recorrido histórico por la educación artística observamos como a lo largo de todo el siglo XX e inicios del siglo XXI se oscila casi siempre entre dos polos de atención básicos para orientarla: el individuo o la sociedad. Según la prioridad que se dé a uno u otro aspecto surgen propuestas atentas: o bien, a la formación del alumno, a su libertad y autonomía, o bien, a la transformación social, a la mejora de la educación y de la calidad de vida de las clases más desfavorecidas o a la crítica social. En la actualidad, como demuestra el modelo educativo finlandés de EA desde 1998, o como propugna la UNESCO en sus documentos guía de cara al futuro (Hoja de Ruta y Agenda de Seúl) se ha producido una cierta aproximación e integración entre ambas posturas al defenderse propuestas centradas en la formación individual de los alumnos pero atentas, a la vez, a potenciar la equidad social y a desarrollar capacidades sociales necesarias para una ciudadanía democrática, responsable y activa, que a la vez sepa adaptarse al contexto actual de cambios económicos, culturales y sociales. Junto a estas posturas de considerar el Arte y la creación artística como un beneficio para lo humano y lo social, se produce una revalorización de la educación artística como disciplina imprescindible en el currículum escolar y para la formación permanente de todos los ciudadanos a lo largo de su vida.

\section{La educación de las personas con diversidad funcional}

Resaltamos el discurso de Alexander Jollien para presentar el siguiente campo de estudio. Tal como lo describe en el prefacio del libro Ruedi Imbach, profesor de filosofía de la Universidad helvética de Friburgo, es «un joven suizo con parálisis cerebral que, debido a su hándicap, estaba destinado a liar puros en un taller ocupacional para personas discapacitadas. Ahora, después de un largo periplo, estudia filosofía en la universidad.» (Jollien, 2001:9). Jollien reconoce su deuda con los filósofos que le han ayudado a progresar y a descubrir en el corazón de la debilidad la grandeza del hombre: «... me ha parecido que la ausencia de prejuicios, que por lo general asociamos a Sócrates, le convertía en un excelente compañero de ruta...» (Jollien, 2001:16)

SOCRATES

¡No nos precipitemos!

ALEXANDRE

Una atetosis

SOCRATES

¡Habla más claro!

ALEXANDRE

Como puedes comprobar, me cuesta coordinar los movimientos, camino tambaleándome y hablo con lentitud. Son secuelas de una asfixia que científicamente se conoce con el nombre de atetosis. SOCRATES

¿Y cuál fue la causa?

ALEXANDRE

Por querer hacer demasiadas piruetas dentro del vientre de mi madre, el cordón umbilical se enrolló alrededor del cuello y... tú mismo puedes constatar los destrozos causados. Nací en una atmósfera bastante crítica. Mi madre me explicó cómo vio salir de su vientre a un bebé totalmente negro que no lloraba. “¿Está muerto? “, exclamó. A la enfermera sólo se le ocurrió responder: “No, pero no sabemos si va a tener un buen fin". Por unos instantes, en los ojos fatigados de mi madre se fijó la visión del recién nacido, rápidamente madre e hijo fueron separados. Me llevaron a un hospital especializado en reanimación. (Jollien, 2001:21-22) 
Al leer las reflexiones de Jollien, se descubre el gran interés de su mirada sobre el tema de la DF. Habla desde la persona que es considerada diversa, desde sus propias experiencias, desde el ser diferente, desde el haber vivido 17 años en un centro especializado para personas con parálisis cerebral. La gran mayoría de ensayos sobre el tema están escritos por teóricos, profesionales de la educación especial, que están fuera del ser considerado diferente. Estos expertos en el tema investigan las características de estas personas, sus necesidades y apoyos, sus intereses, su reeducación... sin, a veces, tener verdaderamente presente cómo ellos se sienten, cómo les gustaría que fuera su vida, dónde se encuentra la diversidad y que apoyos necesitan. A diferencia de estos autores, Jollien ha vivido toda su vida en el interior de un cuerpo que no le hace caso y que es diferente al del resto de personas que le han rodeado, la mayoría de las cuales no han entendido sus verdaderas necesidades.

La propia experiencia de Jollien apoyaría el entender a la persona con DF, como una persona con características personales que hacen que necesite de una serie de apoyos específicos para su desarrollo cognitivo, personal y social. La utilización de más o menos apoyos estará condicionada por la interacción que establezca la persona con DF y el entorno en el que viva y se desarrolle a nivel educativo, familiar y social.

SÓCRATES

Una vez más, el miedo a todo aquello que es diferente.

ALEXANDRE

La diferencia trastorna, desconcierta al hombre en su búsqueda por la perfección. Por otra parte,

el miedo lo achica... (A. Jollien, 2001:82-83)

Del mismo modo que en la perspectiva aportada por Jollien, la Organización Mundial de la Salud (OMS) define la DF como resultado de la interacción entre las limitaciones de estas personas y las variables ambientales que influyen en el desarrollo de estas: el entorno, las situaciones sociales y los apoyos.

Así, de esta manera, la DF no está fijada, es fluida, continua y cambiante, depende de las limitaciones funcionales del sujeto y de los apoyos disponibles en el entorno personal. Intervenir o proveer de servicios y de apoyos, que se centren en la conducta adaptativa del individuo, es una forma de reducir las limitaciones funcionales y, por tanto, mejorar la vida de la persona. Conviene precisar que a la hora de elegir los apoyos que se necesitan, se han de tener en cuenta cuatro dimensiones: el funcionamiento intelectual, habilidades cognitivas; las consideraciones psicológicas/ emocionales; las consideraciones físicas/de salud/etiológicas y las consideraciones ambientales.

Concretando más, al utilizar los términos personas con DF, nos estamos refiriendo a aquellos individuos que poseen limitaciones significativas tanto en el funcionamiento intelectual como en la conducta adaptativa. Son personas con unas características individuales tales que necesitan unos apoyos específicos externos para poder desarrollarse como personas el máximo de independientes y autónomas posibles. Estos individuos serán más o menos discapacitados en relación a la posesión (o no) de estos apoyos, de lo cual se deduce que la sociedad y la educación tienen un papel primordial. Así, la educación deberá potenciar todas las capacidades del sujeto en un ambiente normalizado, y la sociedad deberá ser sensible a la realidad de estas personas (a sus características, 
necesidades y apoyos), deberá reconocerle el derecho absoluto a la participación en la comunidad, a una accesibilidad universal, y a una vida de calidad.

La convicción de que la discapacidad no es ni fija ni dicotomizada; antes bien, es fluida, continua y cambiante, dependiendo de las limitaciones funcionales de una persona y de los apoyos disponibles en el entorno del individuo. La demostración de que se reducen las limitaciones funcionales y, por lo tanto, la discapacidad de una persona al proporcionarles intervenciones o servicios y apoyos que se centran en la conducta adaptativa y en el estatus de los roles. Una nueva concepción de la discapacidad que implica comprender que la discapacidad de una persona es el resultado de la interacción entre la persona y su entorno. Una visión transformada de lo que representan las posibilidades vitales de las personas con discapacidad. Esta visión incluye una especial atención a la autodeterminación, la inclusión, la equidad y las fortalezas. Un paradigma de apoyos que subyace a la provisión de servicios a personas con discapacidad y se centra en la vida con apoyo, empleo y educación inclusiva. Una interrelación del concepto de calidad de vida con las técnicas de mejora de la calidad, la garantía de calidad, la gestión de calidad y la evaluación basada en los resultados. (Schalock, 2001:83)

Según Schalock (1999), todas las personas tendrían que conseguir ser auto-determinadas. La auto-determinación es una de las ocho dimensiones que se consideran necesarias para que una persona tenga una buena calidad de vida. El término auto-determinación proviene del término inglés self-determination, cuya traducción sería la propia determinación, la determinación de uno mismo, la auto-determinación. Analizando el concepto, su significado sería que los individuos determinen con precisión lo que desean, que tengan decisión propia sobre su vida, que elijan por ellos mismos lo que más les conviene. La auto-determinación hace referencia, pues, a situaciones relacionadas con la independencia, la libertad y la autonomía.

Todavía hay personas con DF que viven o han vivido en contextos pobres de estímulos (instituciones segregadoras), en los cuales conviven con su propia enfermedad y la de los otros, con una reducción importante de modelos, de relaciones sociales y de experiencias que estimulen su formación. Estas personas han estado inmersas en situaciones pasivas y monótonas en las cuales otros decidían por ellos, en situaciones en las cuales tenían que aceptar todo lo que los demás habían organizado, en situaciones en las cuales los espacios, el tiempo, los objetos no eran referenciales de su propia decisión y no pertenecían a su propia vida. En estos lugares ha sido difícil encontrar ocasiones para poder desarrollar sus potencialidades cognitivas y afectivas, y para poder llegar a tener un conocimiento amplio de ellos mismos en relación con los demás, de sus limitaciones, pero también de sus capacidades, y consecuentemente llegar a desarrollar los componentes para tener una conducta auto-determinada. Así, la auto-determinación constituye una de las dimensiones esenciales de la calidad de vida de todas las personas y uno de los contenidos de las nueve áreas de apoyo a los individuos con DF que contempla la definición de la AAMR (American Association on Mental Retardation) del 2002.

Según Wehmeyer, la auto-determinación representa «la capacidad de actuar como agente causal en la propia vida y elegir y tomar decisiones en relación con la calidad de vida de uno mismo y que estén libres de influencias o de interferencias indebidas.» (Wehmeyer, 2003:80). La conducta auto-determinada se refiere a aquellas acciones que se identifican por cuatro características esenciales: la persona actúa de una manera autónoma, la conducta es autorregulada, la persona inicia y responde a los acontecimientos de una manera psicológicamente hábil y, por último, la 
persona actúa conscientemente.

El concepto de agente causal, al que hace referencia Wehmeyer en su definición, implica que es la persona quien hace o provoca que las cosas pasen en su vida. Las personas con autodeterminación actúan como agentes causales de sus vidas. Ésta irá surgiendo a medida que las personas aprenden determinadas habilidades y desarrollen ciertas actitudes que les permitirán ser los agentes causales de sus vidas. Las habilidades, a las cuales se refiere este autor, son los elementos componentes de la auto-determinación (Wehmeyer, 2006): habilidades para elegir, habilidades de resolución de problemas, habilidades para tomar decisiones, habilidades para establecer y conseguir objetivos, habilidades de autocontrol y autorregulación, habilidades de autodefensa y liderazgo, percepción de control y eficacia y autoconsciencia/autoconocimiento.

Nirje realza la importancia de la auto-determinación para las personas con diversidad funcional:

... una faceta principal del principio de normalización es la de crear condiciones a través de las cuales una persona discapacitada experimente el respeto normal al que cualquier ser humano tiene derecho. Por lo tanto, las elecciones, deseos y aspiraciones de una persona discapacitada deben tomarse en consideración tanto como sea posible en acciones que le afecten. Afirmarse en el seno de la familia, los amigos, los vecinos, los compañeros de trabajo o agentes es una tarea difícil para numerosas personas. Esto es especialmente difícil para alguien que tenga una discapacidad o sea percibida de forma devaluada. Pero al final, incluso la persona disminuida debe seguir adelante como individuo distinto y, por lo tanto, posee su identidad definida por sí mismo y por los otros a través de circunstancias y condiciones de su existencia. Por lo tanto, el camino hacia la autodeterminación es tan difícil como importante para una persona disminuida. (Nirje, cit. en Wehmeyer, 2001:8)

Nirje compara la auto-determinación con el respeto y dignidad a los que tienen derecho todos los seres humanos.

\section{Investigación-acción: diseño, proceso y resultados}

Después de describir, analizar y situarnos en la complejidad de ambos términos: la creación artística y las personas con DF, presentamos la parte empírica de esta investigación.

El objetivo general es el estudio de un taller de creación artística en el que participaron 5 niños y una niña con DF durante un curso escolar. Se analizan las posibilidades de la educación, desde la creación artística, para evidenciar que en estos espacios se desarrollan los componentes de la conducta auto-determinada. Se concretan las características de los espacios de creación artística y se analizan los procesos de creación de los niños, que facilitan el desarrollo de habilidades personales y sociales de los participantes. De esta manera, se podrá justificar la importancia de la creación artística en el desarrollo de la auto-determinación y la mejora de la calidad de vida de las personas con DF.

Como anunciamos ya en el título del texto, recorremos a la investigación-acción como paradigma de análisis: la investigación-acción es una forma de indagación introspectiva colectiva, emprendida por participantes en situaciones sociales con objeto de mejorar la racionalidad y la justicia de sus prácticas sociales o educativas, así como la comprensión de esas prácticas y de las 
situaciones en que éstas tienen lugar (Kemmis y McTaggart, 1988). La investigación-acción es una cuestión abierta, no empieza con una hipótesis sino con una idea inicial y una visión del recorrido y resultados del proceso, que serán evidenciados a lo largo de la investigación.

Stenhouse (1998, en Latorre, 2003), es promotor de la figura del maestro como investigador, argumenta que el cuerpo docente y la educación no deben ser objeto de investigación de personas externas, sino investigador de sí mismo. De igual modo, Dewey (1933, en Latorre, 2003) destaca la importancia de que el profesorado reflexione sobre su propia práctica e integre sus observaciones en las teorías que emergen de los procesos de enseñanza y aprendizaje. El pensador americano de inicios del siglo XX defiende que el profesorado sea al tiempo consumidor y generador de conocimiento: investigador, reflexivo, crítico e innovador de su práctica educativa. Desde nuestra experiencia profesional docente, asumimos estos planteamientos del modelo de investigación en el ámbito educativo descrito; consideramos que existen numerosos agentes internos y externos relacionados con la dinámica escolar, que son muy difíciles de controlar si la presencia del investigador en la escuela es sólo de unas horas o unos días determinados para la realización de la investigación.

La profesión docente está en continuo desarrollo de nuevos conocimientos en relación a los cambios continuos que tienen lugar en la sociedad. La investigación-acción, a la vez que metodología de investigación para resolver problemas educativos, es un modelo también de formación continua y de reflexión para el auto-desarrollo del profesorado. La finalidad será mejorar, innovar, comprender los contextos educativos, teniendo como meta la calidad de la educación, gracias a una investigación en la escuela y desde la escuela.

El ciclo de la investigación-acción se configura en torno a cuatro momentos o fases: planificación o plan de acción, acción, observación y reflexión. La observación, la recogida y análisis de los datos de una manera sistemática y rigurosa otorga al proceso el rango de investigación.

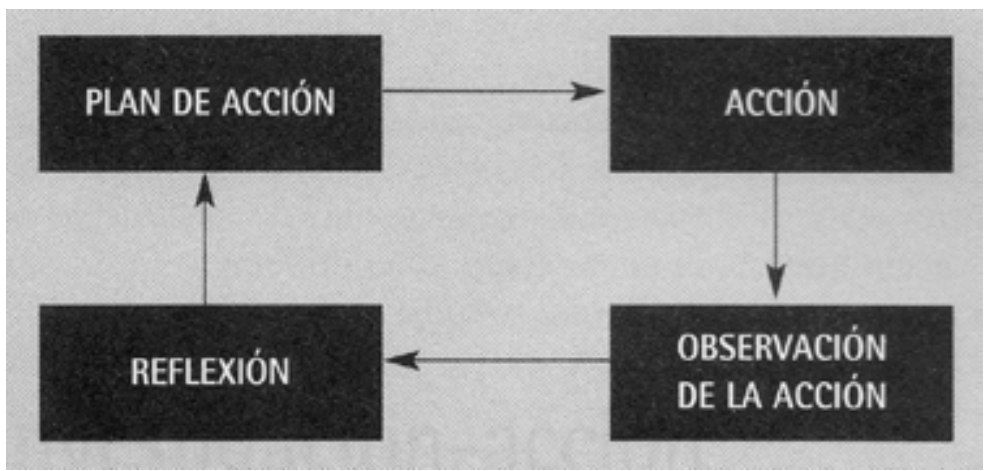

Figura 1. Ciclo de investigación-acción según Latorre (2003:21)

Según Kemis y McTaggart (1988), la finalidad intrínseca del proceso de indagación se organiza siguiendo varios aspectos:

- Aumentar el conocimiento: la investigación-acción produce nuevas teorías o permite reformularlas. 
- Mejorar la actividad: el análisis y la reflexión sistemática se dirigen a mejorar la calidad de la acción, cambiar y transformar lo que se considere para perfeccionar la práctica.

- Interpretar lo que está sucediendo: la dimensión social y ética de la investigación-acción permite a sus protagonistas participar en la comprensión dinámica de la situación (de sus intenciones, expectativas, etc.) para transformarla.

En este trabajo de investigación, tal como está diseñado, se pretende abarcar estos procesos: aumentar o reformular el conocimiento y mejorar la actividad a partir de la interpretación de lo que sucede.

Siguiendo el ciclo de la investigación-acción según Whitehead (1989, en Latorre, 2003:38), nuestra investigación-acción se estructura de la siguiente manera:

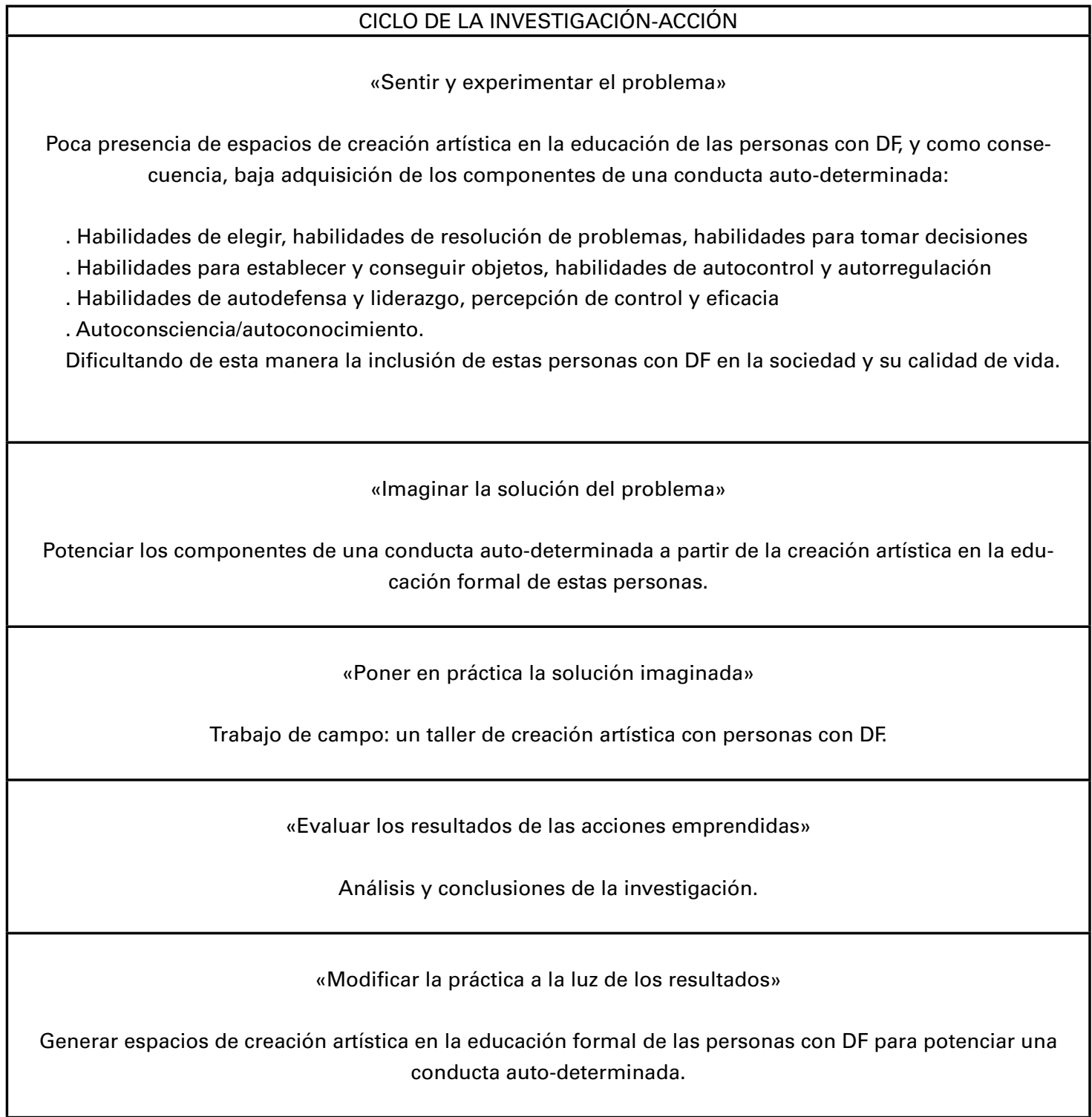

Tabla 1. Estructura de la investigación-acción 
La investigación se lleva a cabo con un grupo de cinco niños y una niña con DF y se sigue el ciclo antes presentado. Se propusieron 22 sesiones de creación artística, en los que tomo el rol de maestra-investigadora durante un curso escolar.

El espacio-tiempo para la creación artística ha de tener las características ${ }^{5}$ que definimos posteriormente para conseguir los resultados esperados:

- Una atmósfera amable de aceptación personal de cada alumno -pese a algunas conductas disruptivas- y de aceptación de las acciones y producción de cada uno de los participantes. Esta atmósfera dura toda la secuencia y es considerada indispensable tanto para la creación como para el desarrollo de la auto-determinación.

- Relación de confianza entre los participantes. Se va intensificando a medida que avanzan las sesiones y se observa que los comportamientos se van haciendo más desinhibidos. Los alumnos son capaces de pedir ayuda a las maestras y de proporcionar ayuda a compañeros.

- Respeto al ritmo e intereses de cada participante.

- Espacio de libertad y seguridad. Afloran sentimientos y emociones profundas, tanto positivas como negativas: la frustración y la rabia, y la desorientación ante el mundo adulto. Se observa que conductas disruptivas, consecuencia de estas emociones negativas, van disminuyendo a lo largo del curso.

- Las sesiones de taller siguen la siguiente estructura:

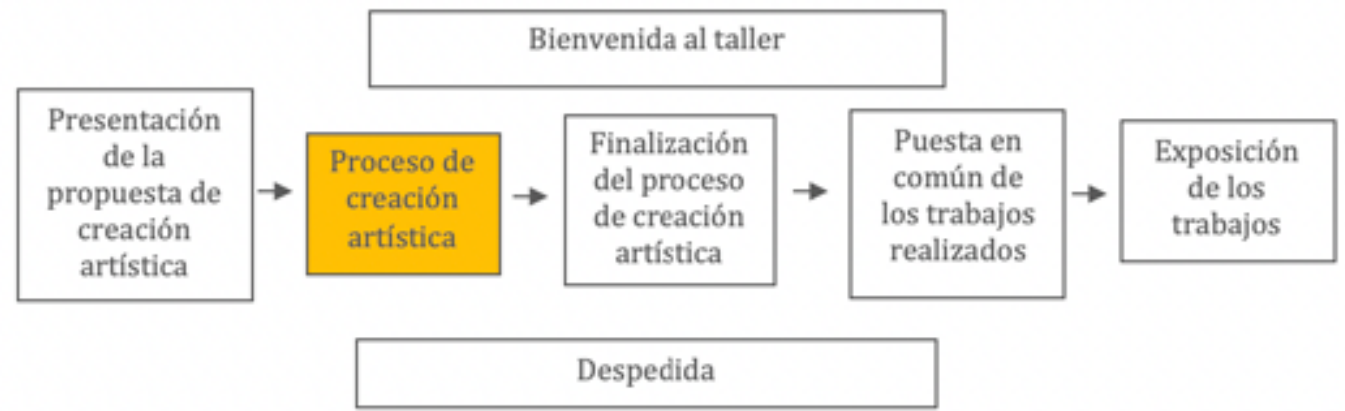

Figura 2. Estructura de las sesiones de taller

- Procesos de creación y resultados únicos, pensados y decididos por cada participante. Se van haciéndose conscientes de su individualidad, van conociendo sus capacidades y limitaciones desde las experiencias y la reflexión. Van construyendo su propia identidad dentro del grupo, un auto-conocimiento en paralelo al conocimiento de los otros y del mundo.

- Disposición de materiales fungibles y no fungibles para el uso autónomo y responsable.

- Un tiempo y un espacio donde poder circular de manera autónoma y libre.

5 Estas prácticas educativas habían sido ensayadas previamente por la maestra-investigadora. El recorrido de la investigación permitió concretar las características de los espacios de creación artística. 
- Propuestas abiertas, que dan la posibilidad de reformularlas a partir de los intereses y posibilidades de cada participante.

- Espacio que permita la experimentación libre y la exploración.

- Un lugar de comunicación libre e interacción a través de los lenguajes artísticos, que ofrezca posibilidades para percibir, experimentar, inventar, decidir, descubrir, imaginar, crear de manera autónoma. La experimentación con los lenguajes artísticos ofrece recursos para aumentar y mejorar la comunicación. Durante la secuencia, se utiliza el lenguaje musical, el corporal y el lenguaje visual y plástico.

- La elección personal y la toma de decisiones individuales como conductoras de los procesos de creación. Los participantes eligen sus propios temas para la creación individual y participan con sus propias ideas en las creaciones colectivas. Se potencia la iniciativa, la creatividad y la responsabilidad ya que son los propios alumnos los que van concretando y planificando todos los detalles de sus proyectos.

- Tareas semi-dirigidas que transitan hacia trabajos auto-dirigidos. Los participantes conducen la evolución del contenido temático de la creación artística en función de los logros y conveniencias que van surgiendo.

- Espacios que potencien la recompensa intrínseca.

- La maestra se muestra como acompañante-guía y modelo de actuación con actitud de proximidad emocional. Su intervención constituye un estímulo para la ejecución del niño, nunca proporciona una instrucción directa.

- Se estimula la capacidad para descubrir el gusto por indagar, por las cosas desconocidas, incluso cuando ello exige esfuerzo y concentración.

Cada sesión-taller constituye una aventura en la que observar, experimentar, descubrir, pensar, proyectar, crear, inventar, relacionarse, etc.

\subsection{Niveles de análisis}

\subsubsection{Recorrido por la secuencia didáctica. Poner en práctica la solución imaginada}

El objetivo de este primer nivel de análisis es hacer un recorrido por la secuencia didáctica a partir de la presentación y análisis de cada sesión y de los comentarios sobre el proceso de enseñanzaaprendizaje desde una perspectiva transversal. Durante este trayecto se ha hecho incidencia en comportamientos que corresponden a los diferentes componentes de la auto-determinación: habilidades que tenían relación con la autonomía personal (independencia), habilidades para elegir, para resolver problemas, para tomar decisiones, para establecer y conseguir objetivos, de autocontrol y autorregulación, de autodefensa y liderazgo, de percepción de control y eficacia y, por último, de autoconciencia y de autoconocimiento.

A través de este primer nivel de análisis se obtiene una descripción de la secuencia didáctica del taller de creación artística con sus participantes, el contenido de las sesiones y estructura. Se puede apreciar como los temas cambian en función de los intereses de los participantes y como 
se concretan a partir de un momento determinado en torno a un proyecto: «el circo».

Los análisis muestran como el aula-taller se va construyendo como contexto educativo-creativo a lo largo de la secuencia didáctica entre todos los participantes. Los alumnos van conociendo los materiales, sus características y utensilios específicos para trabajarlos. Van conociendo su lugar en el aula-taller; van siendo progresivamente autónomos e independientes. La realización de una actividad común, la participación para la construcción de un todo, es un ejemplo de comportamiento auto-determinado por parte de los alumnos: actúan autónomamente, resuelven problemas, deciden, eligen, se auto-gestionan, etc. y realizan, también en este caso, un trabajo cooperativo. Se benefician de lo que los lenguajes artísticos tienen de únicos: un canal de expresión de la parte más íntima y personal.

Se puede concluir que el proceso cumple con las expectativas creadas inicialmente por el proyecto. Se puede comprobar cómo algunos de los componentes de la autodeterminación son requeridos y tienen ocasión de manifestarse y de desarrollarse.

\subsubsection{Segundo nivel de análisis: Evaluar los resultados de las acciones emprendidas}

En el segundo nivel de análisis se presentan los comportamientos de autodeterminación observados a lo largo de la secuencia didáctica agrupados según la tipología de componentes de la autodeterminación que concreta Wehmeyer (2006). Se destacan tres comportamientos a modo de indicadores de auto-determinación y se analiza su presencia, su frecuencia y la forma que toman a lo largo de la secuencia para todo el grupo y para cada uno de los 6 alumnos que componen el grupo clase objeto de la intervención.

Con este segundo nivel de análisis se concretan 90 tipos de comportamientos diferentes que corresponden a los componentes de autodeterminación de la tipología de Wehmeyer (2006) observados a lo largo de la secuencia didáctica de creación artística.

El componente que destaca con mayor número de tipos de comportamiento identificados es el de auto-regulación/auto-control. Ello nos lleva a la reflexión de que en un contexto educativo y de creación artística los alumnos pueden alcanzar niveles altos de auto-regulación sin depender de pautas constantes del adulto y sin necesitar refuerzos inmediatos. En un planteamiento como el de la secuencia analizada la recompensa intrínseca es un elemento esencial del proceso.

El análisis nos ha llevado a considerar que todos los componentes de la auto-determinación se hayan estrechamente relacionados en la actividad. En realidad, funcionan como un sistema en el que destacan dos que engloban a los demás: las habilidades para la resolución de problemas y para establecer y conseguir objetivos. Elegimos 3 indicadores de alto nivel de auto-determinación para profundizar en el análisis:

- ser capaces de comunicar objetivos y propuestas ya sea por iniciativa propia, por copia de un compañero y en menor medida a propuesta de la maestra o de un compañero;

- lograr una parte de esos objetivos planificados, y

- resolver problemas autónomamente. 
Los indicadores han sido identificados, aunque su presencia no aumenta cuantitativamente a lo largo de la secuencia. Sin embargo, paralelamente se aprecia un aumento de comportamientos relacionados con la auto-determinación, los alumnos se mueven libremente por el espacio del taller persiguiendo sus objetivos sin pedir ayuda durante largos periodos de tiempo, lo que se hace más visible a medida que avanza la secuencia. Los alumnos tienen ideas y son capaces de proponer cosas cada vez más complejas y que afectan a todo el grupo (hacer una carpa, una orquesta, etc.). Ello nos lleva a la reflexión de que quizás la elección de indicadores no fue acertada porque su identificación depende excesivamente de la verbalización. Si bien son representativos de auto-determinación, los tres indicadores corresponden a conductas de nivel de exigencia excesivo para el caso analizado. Se trata de 6 alumnos con limitaciones importantes, con edades cronológicas entre 7 y 12 años y edad mental sensiblemente inferior, acostumbrados a una metodología tradicional en el sentido de pautada y rutinaria, basado en el enfoque de reeducación de habilidades, más que de estimulación de competencias.

Pese a ello, los indicadores parecen útiles a la hora de ayudar a la planificación docente, ya sea para detectar problemas en el proceso por parte de los alumnos que les lleva a la autodeterminación (con lo cual se pueden realizar reajustes docentes dentro de la secuencia), ya sea para comprobar la idoneidad de las situaciones que se plantean para generar este tipo de aprendizajes. La limitación señalada anteriormente, de depender excesivamente de la verbalización, es a la vez garantía de objetividad, ya que puede señalarse inequívocamente o no la presencia de dichas conductas en base a sí existe o no conducta verbal indicadora (otra cosa es la conducta autodeterminada de este tipo no verbalizada, que no queda recogida en este análisis).

Podemos concluir que todos los alumnos son capaces de comunicar objetivos y propuestas ya sea por iniciativa propia, por copia de un compañero y en menor medida a propuesta de la maestra o de un compañero. Logran una parte de esos objetivos. El comportamiento que aparece en menor medida es el de identificar y resolver problemas autónomamente.

En suma, y a pesar de las limitaciones de la intervención, se aprecian conductas características de la auto-determinación en todos los alumnos, aunque también se aprecian importantes diferencias entre ellos.

\section{Discusión de resultados y conclusiones. Modificar la práctica a partir de los resultados}

Como se desprende de nuestro trabajo, la creación artística (en todos sus lenguajes) potencia los componentes de la auto-determinación por lo que será imprescindible que forme parte de la formación de todos los niños y niñas con DF. Para ello, los/as maestros/as han de desarrollar sus capacidades creativas y de creación para poder acompañar a sus alumnos en estos procesos que desarrollan y afianzan los comportamientos que muestran una conducta auto-determinada y que corresponden a habilidades básicas para la participación activa en la sociedad, y en consecuencia, una mejora en el proceso de inclusión y en la mejora de la calidad de vida. Es imprescindible, por 
tanto, que en la educación de las personas con DF se creen espacios de libertad y seguridad en los que se puedan expresar, experimentar los miedos y el goce del logro de la creación, así como intercambiar y contrastar entre iguales los propios criterios y las estrategias que la hacen posible.

La práctica de la creación artística debe incluir la reflexión sobre los procesos personales de creación, como elemento que ayuda a conceptualizar los elementos artísticos y a acrecentar las propias habilidades.

En cuanto al impacto de la intervención en la comunidad de práctica y a los procesos reflexivos a que ha dado lugar (Dewey, 1974; Schon, 1992), cabe destacar que, con posterioridad a la misma, la dirección del centro propuso a la maestra-investigadora la realización de un curso de formación permanente sobre educación artística para el equipo docente del centro. El asesoramiento consistió en presentar proyectos de creación artística llevados a cabo por la profesora en el ámbito de la educación inclusiva y además en la realización de procesos de creación artística por parte de las maestras participantes, seguida de los análisis correspondientes. A través de ese proceso de formación, llegaron a crear conocimiento sobre la práctica artística y el proceso de creación desde sus propias experiencias: investigaron, descubrieron, eligieron, pensaron, crearon, etc. Se les dio la oportunidad de un tiempo y un espacio de libertad de creación acompañados por una especialista que, desde una proximidad afectiva, les ofrecía confianza y seguridad. Las maestras observaron cómo se potenciaban habilidades relacionadas con la auto-determinación desde su propia experiencia. Estos espacios y tiempo de creación en común indujeron a nuevas interacciones entre los participantes, que ayudaron al conocimiento de los otros y así, valorar las capacidades y competencias de cada miembro, mejorando el trabajo en equipo.

El área de educación artística toma otro enfoque en la escuela desde esta experiencia: el equipo de maestras decidió cambiar la planificación del centro y nombrar a una profesora como especialista del área de visual y plástica, así como reconvertir un aula en taller de creación artística en el que participan todos los alumnos del centro (actualmente, todavía funciona).

Otro impacto de la investigación fue el crear espacios de creación artística en el espacio museístico que tenían como principal objetivo: acercar el arte y la cultura a personas con DF y fomentar los componentes de una conducta auto-determinada. La maestra-investigadora de este trabajo diseñó y condujo un primer proyecto de Educación Artística para personas con DF, Ments Creatives, permitiendo llevar a la práctica la solución imaginada de la investigación en el ámbito del museo. Este proyecto de EA se inicia con un trabajo de observación, reflexión y análisis; y de investigación y creación a partir de la contemplación directa de las obras de arte, donde se facilita a los participantes la experiencia de vivir un espacio y un tiempo dedicado a la creación artística: un espacio amable de libertad con seguridad, en el que los participantes pueden investigar, decidir, descubrir, imaginar, elegir, crear, etc. Posteriormente, se realizaron dos proyectos más con las mismas características: La ciutat: un lloc per viure y Retrats. Todos forman parte del Programa $\mathrm{Mu}$ seu Un espai per a tothom, del Museo Nacional de Arte de Catalunya. Se consideran proyectos de educación artística, disciplina que contempla, como bien nos dice Eisner (2005), cuatro ámbitos principales: la historia del arte, la estética, la crítica y la creación artística.

Todos estos proyectos han finalizado con una exposición del proceso y de los resultados en 
el museo abierta al público y como explica Teresa González ${ }^{6}$ en el tríptico informativo de la primera exposición, la creación artística es imprescindible para la educación de todas las personas, incluidas aquellas que forman parte de colectivos en riesgo de exclusión social, como las personas con DF:

Los museos son espacios de interacción y de relación con una fuerte identidad cultural que pueden contribuir al desarrollo de sentimientos de pertenencia, respeto, tolerancia, dignidad y solidaridad social. Son lugares donde ejercitar la mente, expresar ideas y emociones, donde desarrollar la creatividad y la imaginación. Son también entornos de aprendizaje que dan la oportunidad de acceder a la educación y a la cultura; lugares de inspiración y de diversión; espacios que permiten el desarrollo de la autonomía personal, la auto-estima y la confianza y que invitan a comunicarnos, relacionarnos y a participar. En este escenario y bajo estas premisas ha tenido lugar la experiencia que presentamos en esta muestra y que tiene como objetivo principal hacer visible y dar a conocer a la sociedad la vertiente creativa de aquellas personas con riesgo de sufrir exclusión social o cultural. A la vez, es, también, uno recopilatorio documental de la experiencia y de los protagonistas, de los procesos que ha habido y de sus resultados. La muestra se convierte, así, en un intercambio de miradas que dialogan las unas con las otras. La mirada que sobre el patrimonio artístico han hecho todas las personas que han participado, la del artista-comisario que construye una nueva mirada a partir de la documentación del proyecto y finalmente el intercambio de miradas que se establece entre, vosotros, espectadores de la muestra, y cada uno de sus protagonistas. ${ }^{7}$ (González, 2007)

\section{Referencias bibliográficas}

AAMR (2006). El retard mental. Definició, classificació i sistemes de suport. Barcelona: Eumo Editorial.

Dewey, J. (1933). How we think. Boston: Health \& Co (Traducción castellana: Cómo pensamos. Barcelona: Paidós, 1989.

Dewey, J. (1974). John Dewey on education: Selected writings. Chicago: University of Chicago Press. Dewey, J. (2008). El arte como experiencia. Barcelona: Paidós.

Eisner, E. (2005). Educar la visión artística. Barcelona: Paidós.

Ferrés, J. (2008). La educación como industria del deseo. Un nuevo estilo comunicativo. Barcelona: Gedisa.

Freire, H. (2008). Arte infantil y transformación social. El rapto de Europa. Pensamiento y creación, 13, 23-38. Madrid: Calamar Edición.

Jollien, A. (2001). Elogio de la debilidad. Barcelona: RBA S.A.

Kemmis, S., y Mc Taggart, R. (1988). Cómo planificar la investigación-acción. Barcelona: Laertes.

Nirje, B. (1972). The right to self-determination. En W. Wolfensberger (Ed.), Normalization: the principle of normalization, pp. 176-200. Toronto: National Institut on Mental Retardation.

Schalock, R. L. (1999). Hacia una nueva concepción de la discapacidad. Siglo Cero. Revista española sobre discapacidad intelectual, 30, 1.

6 Teresa González, responsable del Departament d'Educació del MNAC, especialista en educación y museos. En el MNAC llevan a cabo programas para personas con DF desde el año 1999 y actualmente, están trabajando para que su programación sea accesible a todo tipo de público. Los programas incluyen la formación de los educadores tanto los del museo como los de los centros participantes en sus actividades/proyectos.

7 Tríptico exposición ments creatives, desembre, 2007. Sala Oval del Museu Nacional d’Art de Catalunya. 
Schalock, R. L. (2001) Conducta adaptativa, competencia personal y calidad de vida. En A. Verdugo y F. Borja (Coords.), Apoyos, autodeterminación y calidad de vida. IV Jornadas científicas de investigación sobre personas con discapacidad, pp. 83-104. Salamanca: Amaru Ediciones.

Schon, D. A. (1992). La formación de profesionales reflexivos. Hacia un nuevo diseño en la enseñanza y el aprendizaje en las profesiones. Madrid: Paidós/MEC.

Stenhouse, L. (1998). La investigación como base de la enseñanza. Madrid: Morata.

UNESCO (2006). Conferencia Mundial sobre la Educación Artística: construir capacidades creativas para el siglo XXI. Hoja de ruta para la Educación Artística. Recuperado el 2/02/2019, de: http://portal.unesco.org/culture/es/ev.php-URL ID=39546\&URL DO=DO TOPIC\&URL SECTION=201.html.

UNESCO. (2010) La Agenda de Seúl: Objetivos para el desarrollo de la educación artística. Recuperado el 2/02/2019, de: http://portal.unesco.org/culture/es/ev.php-URL $\underline{I D=2916 \& U R L ~ D O=D O ~ T O P I C \& U R L ~ S E C T I O N=201 . h t m l . ~}$

Wehmeyer, M. L. (2001). Autodeterminación una visión de conjunto conceptual y análisis empírico. Siglo Cero. Revista española sobre la discapacidad intelectual, 32(2),194, 5-15.

Wehmeyer, M. L. (2003). Autodeterminació i accés al currículum general. Promoure l'autodeterminació i l'accés al currículum general. Suports, 7(2), 78-90.

Wehmeyer, M. L. (2006). Autodeterminación y personas con discapacidades severas. Siglo Cero. Revista española sobre Discapacidad Intelectual, 37(4), 220, 4-16.

Whitehead, J. (1989). How do we improve research-based professionalism in education? A question which includes action research, educational theory and the politics of educational knowledge. British Educational Research Journal, 15(1), 3-17. 